\title{
One-carbon homologation of aryl and alkyl aldehydes to amides using $\mathrm{BtCH}_{2} \mathrm{P}^{+} \mathrm{Ph}_{3} \mathrm{Cl}^{-}$
}

\author{
Alan R. Katritzky,* Rong Jiang, Geoffroy L. Sommen, and Sandeep K. Singh \\ Center for Heterocyclic Compounds, Department of Chemistry \\ University of Florida, Gainesville, FL 32611-7200 \\ E-mail: Katritzky@chem.ufl.edu
}

Dedicated to Professor Chengye Yuan on his $80^{\text {th }}$ anniversary

(received 21 Apr 04; accepted 28 May 04; published on the web 03 June 04)

\begin{abstract}
One-carbon homologated amides have been prepared from aryl and alkyl aldehydes. Vinyl benzotriazoles 6, prepared by the reaction of aldehydes with the one-carbon synthon $\mathrm{BtCH}_{2} \mathrm{P}^{+} \mathrm{Ph}_{3} \mathrm{Cl}^{-}$(5), were subsequently brominated to give 1,2-dibromo-benzotriazolylalkanes 7. Treatment of 7 with a variety of aryl or alkyl amines in the presence of water furnished onecarbon homologated amides 8ãr in 3205\% yields. This method works well with both primary and secondary aryl or alkyl amines.
\end{abstract}

Keywords: Vinyl benzotriazole, 1,2-dibromo-benzotriazolylalkanes, homologation

\section{Introduction}

Previous methods for the one-carbon homologation of aldehydes to amides have mostly involved the one-carbon homologation of aldehydes $\mathbf{1}$ to carboxylic acids $\mathbf{3}$ followed by a functional group transformation to amides 4 (Scheme 1). Classical intermediates developed for the preparation of one-carbon homologated carboxylic acids $\mathbf{3}$ as summarized by Watt et. al. include: cyanohydrins $\mathbf{2 a}$, nitriles $\mathbf{2 b}$, glycidic esters $\mathbf{2 c}$, epoxides $\mathbf{2 d}$, enol ethers $\mathbf{2 e}$, thioenol ethers $\mathbf{2 f}$, enamines $\mathbf{2 g}$, ketene thioacetals $\mathbf{2 h}, \alpha, \beta$-unsaturated sulfones $\mathbf{2} \mathbf{i}$, or $\alpha, \beta$-unsaturated phosphonates $\mathbf{2} \mathbf{j}$ (Scheme 1). ${ }^{1}$ Syntheses of intermediates $\mathbf{2} \mathbf{a}-\mathbf{j}$ and subsequent conversion to carboxylic acids $\mathbf{3}$ often require harsh conditions that are incompatible with sensitive functional groups. Reports of low yields using these intermediates and lack of sufficient examples indicate a limited scope. A one-pot procedure via 1-(benzotriazol-1-yl)-1-methoxyalk-1-enes $\mathbf{2 k}$ for the preparation of homologated carboxylic acids from aldehydes has been reported (Scheme 1). ${ }^{2}$ Amine solvolysis of $\alpha$-alkoxyacrylonitriles $\mathbf{2 l}$ gives one-carbon homologated amides directly but is inapplicable to substrates with acid-labile groups (Scheme 1). ${ }^{\text {la }}$ The recently reported one- 
carbon homologation of aldehydes to amides using 1,1-dibromo-1-alkenes $\mathbf{2 m}$ offers a short route, good yields and mild reaction conditions that tolerate a range of functionalities but it is inapplicable to alkyl aldehydes and aryl amines and uses carcinogenic carbon tetrabromide (Scheme 1). ${ }^{3,4}$

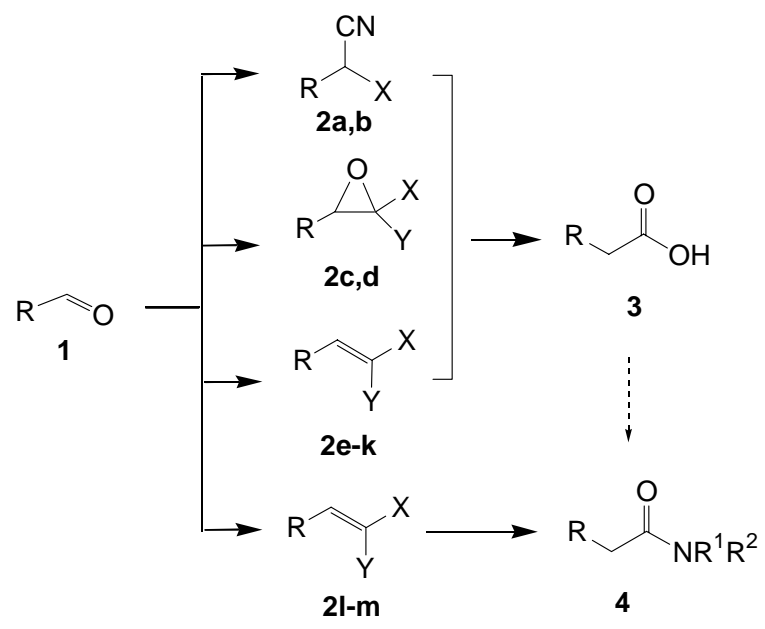

(2a) $\mathrm{X}=\mathrm{OH}$; (2b) $\mathrm{X}=\mathrm{H}$; (2c) $\mathrm{X}=\mathrm{H}, \mathrm{Y}=\mathrm{COOR}$; (2d) $\mathrm{X}=\mathrm{Y}=\mathrm{H}$; (2e) $\mathrm{X}=\mathrm{H}, \mathrm{Y}=\mathrm{OMe}, \mathrm{OPh}$; (2f) $\mathrm{X}=\mathrm{H}, \mathrm{Y}=\mathrm{SPh} ;(\mathbf{2 g}) \mathrm{X}=\mathrm{N}\left(\mathrm{CH}_{2}\right)_{4}, \mathrm{NMe}_{2} ;(\mathbf{2 h}) \mathrm{X}, \mathrm{Y}=\mathrm{SMe}, \mathrm{SPh}, \mathrm{S}\left(\mathrm{CH}_{2}\right)_{3} \mathrm{~S}$; (2i) $\mathrm{X}=\mathrm{SO}_{2} \mathrm{Ph}$, $\mathrm{Y}=\mathrm{NHCHO} ;(\mathbf{2 j}) \mathrm{X}=\mathrm{PO}(\mathrm{OEt})_{2}, \mathrm{Y}=\mathrm{NMe}_{2} ;(\mathbf{2 k}) \mathrm{X}=\mathrm{Bt}, \mathrm{Y}=\mathrm{OMe} ;(\mathbf{2 l}) \mathrm{X}=\mathrm{CN}, \mathrm{Y}=\mathrm{OAc}$;

$(2 \mathrm{~m}) \mathrm{X}=\mathrm{Y}=\mathrm{Br}$

\section{Scheme 1}

Herein, we report a general method for the conversion of aldehydes to one-carbon homologated amides. Vinyl benzotriazoles 6 prepared from $\mathrm{BtCH}_{2} \mathrm{P}^{+} \mathrm{Ph}_{3} \mathrm{Cl}^{-}(5)$ and aldehydes ${ }^{5}$ were brominated to give 1,2-dibromo-benzotriazolylalkanes 7. Subsequent treatment of 7 with a variety of aryl or alkyl amines in the presence of water furnished the desired one-carbon homologated amides 8a-r in 32-95\% yields. Our method is applicable to aryl and alkyl aldehydes as well as to primary and secondary aryl or alkyl amines and avoids the use of carbon tetrabromide.

\section{Results and Discussion}

Preparation of vinyl benzotriazoles and 1,2-dibromo-benzotriazolylalkanes. Reaction of $\mathrm{BtCH}_{2} \mathrm{P}^{+} \mathrm{Ph}_{3} \mathrm{Cl}^{-}$(5) with $n$-BuLi and aryl or alkyl aldehydes (RCHO, $\mathrm{R}=\mathrm{Ph}$, 2-thienyl, $i$-Pr, Et) gave vinyl benzotriazoles 6a-d in $32-80 \%$ yields following a previously reported general procedure (Scheme 2). ${ }^{5}$ Treatment of vinyl benzotriazoles 6 a-d with bromine at $0{ }^{\circ} \mathrm{C}$ gave the corresponding 1,2-dibromo-benzotriazolylalkanes 7 a-d in 83-100\% yields (Scheme 2). Most of the dibromoalkanes were obtained in quantitative yields and could be used directly in the next step without further purification. 


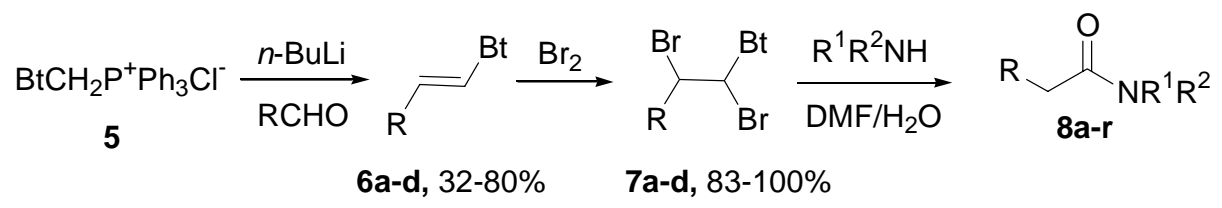

\section{Scheme 2}

Preparation of amides. Reaction of 1,2-dibromo-benzotriazolylalkanes 7 a-d with various alkyl or aryl amines (5 equiv) in $\mathrm{DMF} / \mathrm{H}_{2} \mathrm{O}$ (3:1) furnished the desired amides 8 a-r in 32-95\% yields (Scheme 2, Table 1). The amides 8 a-r were fully characterized by ${ }^{1} \mathrm{H}$ and ${ }^{13} \mathrm{C}$ NMR spectroscopy, melting point and elemental analysis for novel compounds.

Table 1. Preparation of one-carbon homologated amides 8 a-r

\begin{tabular}{|c|c|c|c|c|}
\hline Entry & $\mathrm{R}$ & $\mathrm{R}^{1}$ & $\mathrm{R}^{2}$ & Yield $(\%)$ \\
\hline 1 & $\mathrm{Ph}$ & $\mathrm{Ph}\left(\mathrm{CH}_{2}\right)_{2}$ & $\mathrm{H}$ & $\mathbf{8 a}(47)$ \\
\hline 2 & $\mathrm{Ph}$ & $\mathrm{Bu}$ & $\mathrm{H}$ & $\mathbf{8 b}(58)$ \\
\hline 3 & $\mathrm{Ph}$ & $\left(\mathrm{CH}_{2}\right)_{5}$ & - & $\mathbf{8 c}(61)$ \\
\hline 4 & $\mathrm{Ph}$ & $\mathrm{Ph}$ & $\mathrm{H}$ & 8d (72) \\
\hline 5 & $\mathrm{Ph}$ & $t-\mathrm{Bu}$ & $\mathrm{H}$ & 8e (68) \\
\hline 6 & $\mathrm{Ph}$ & 2-pyridyl & $\mathrm{H}$ & $\mathbf{8 f}(85)$ \\
\hline 7 & $\mathrm{Ph}$ & 1-pyrimidyl & $\mathrm{H}$ & $\mathbf{8 g}(80)$ \\
\hline 8 & 2-thienyl & $\mathrm{Ph}\left(\mathrm{CH}_{2}\right)_{2}$ & $\mathrm{H}$ & $8 h(52)$ \\
\hline 9 & 2-thienyl & $\mathrm{Bu}$ & $\mathrm{H}$ & $8 \mathbf{i}(92)$ \\
\hline 10 & 2-thienyl & $\left(\mathrm{CH}_{2}\right)_{5}$ & - & $8 \mathbf{j}(88)$ \\
\hline 11 & $i-\operatorname{Pr}$ & $\left(\mathrm{CH}_{2}\right)_{5}$ & - & $\mathbf{8 k}(50)$ \\
\hline 12 & Et & $\mathrm{Bu}$ & $\mathrm{H}$ & $81(84)$ \\
\hline 13 & Et & $\left(\mathrm{CH}_{2}\right)_{2} \mathrm{~N}\left(\mathrm{CH}_{3}\right)_{2}$ & $\mathrm{H}$ & $\mathbf{8 m}(32)$ \\
\hline 14 & Et & $\left(\mathrm{CH}_{2}\right)_{2} \mathrm{O}\left(\mathrm{CH}_{2}\right)_{2}$ & - & $8 n(54)$ \\
\hline 15 & Et & $\left(\mathrm{CH}_{2}\right)_{2} \mathrm{~N}\left(\mathrm{CH}_{3}\right)\left(\mathrm{CH}_{2}\right)_{2}$ & - & $80(62)$ \\
\hline 16 & Et & $\mathrm{Ph}$ & $\mathrm{H}$ & $\mathbf{8 p}(95)$ \\
\hline 17 & Et & 4-pyridyl & $\mathrm{H}$ & $\mathbf{8 q}(82)$ \\
\hline 18 & Et & $\alpha$-napthyl & $\mathrm{H}$ & $8 \mathbf{r}(68)$ \\
\hline
\end{tabular}


The reaction of dibromoalkanes 7 a-d with amines in $\mathrm{DMF} / \mathrm{H}_{2} \mathrm{O}$ is similar to the recently reported method by Shen and Kunzer involving the reaction of dibromoalkenes (prepared from aryl aldehydes) with alkyl amines. ${ }^{3}$ The use of benzotriazolyl intermediates 7 a-d extends the applicability of this method and allows the preparation of one-carbon homologated amides from aryl or alkyl aldehydes and amines.

\section{Conclusions}

In summary, we have developed a general method for the preparation of one-carbon homologated amides from aldehydes via easily accessible 1,2-dibromo-benzotriazolylalkanes.

\section{Experimental Section}

General Procedures. Melting points were determined on a MEL-TEMP capillary melting point apparatus equipped with a Flucke 51 digital thermometer. ${ }^{1} \mathrm{H}$ NMR $(300 \mathrm{MHz})$ and ${ }^{13} \mathrm{C}$ NMR (75 MHz) spectra were recorded on a Gemini $300 \mathrm{NMR}$ spectrometer in $\mathrm{CDCl}_{3}$ (with TMS for ${ }^{1} \mathrm{H}$ and chloroform- $d$ for ${ }^{13} \mathrm{C}$ as the internal reference). Elemental analyses were performed on a Carlo Erba-1106 instrument. THF was distilled from sodium/benzophenone prior to use. All of the reactions with air sensitive compounds were carried out under $\mathrm{N}_{2}$. Column chromatography was performed on silica gel 200-425 mesh.

\section{Procedure for the preparation of benzotriazolyl alkenes 6a-d}

To a solution of 1-(1H-1,2,3-benzotrazolyl)-1-triphenyl methane chloride (21.7 g, 0.05 mol) in DMSO, $n$-BuLi $(1.6 \mathrm{M}, 31 \mathrm{~mL}, 0.05 \mathrm{~mol})$ was added under nitrogen at $25{ }^{\circ} \mathrm{C}$. One hour later aldehyde $(0.06 \mathrm{~mol})$ was added dropwise. After stirring for $12 \mathrm{~h}$, the mixture was poured into water $(150 \mathrm{~mL})$, followed by extraction with $\mathrm{CHCl}_{3}(4 \times 50 \mathrm{~mL})$. The combined extracts were washed with brine and dried over anhydrous $\mathrm{MgSO}_{4}$. After removing the solvent in vacuo, the crude product was purified by column chromatography with hexanes/ethyl acetate (10:1) as eluent.

1-[(E)-2-Phenylethenyl]-1H-1,2,3-benzotriazole (6a). White microcrystals; mp 115-116 ${ }^{\circ} \mathrm{C}$ $\left(\mathrm{Lit}^{5} \mathrm{mp} 115-117{ }^{\circ} \mathrm{C}\right)$; yield, 50\%. ${ }^{1} \mathrm{H}$ NMR $\delta$ 7.28-7.62 (m, 8H), $7.74(\mathrm{~d}, J=8.3 \mathrm{~Hz}, 1 \mathrm{H}), 7.92$ $(\mathrm{d}, J=14.6 \mathrm{~Hz}, 1 \mathrm{H}), 8.09$ (d, $J=8.3 \mathrm{~Hz}, 1 \mathrm{H}) .{ }^{13} \mathrm{C}$ NMR $\delta 110.0,120.4,121.0,121.7,124.6$, $126.5,128.2,128.4,128.9,131.4,134.3,146.3$.

1-[(E)-2-(3-Thienyl)ethenyl]-1H-1,2,3-benzotriazole (6b). Yellow powder; mp 80-82 ${ }^{\circ} \mathrm{C}$; yield, 68\%. ${ }^{1} \mathrm{H}$ NMR $\delta 7.04(\mathrm{dd}, J=3.3,5.0 \mathrm{~Hz}, 1 \mathrm{H}), 7.17(\mathrm{~d}, J=3.3 \mathrm{~Hz}, 1 \mathrm{H}), 7.27(\mathrm{~d}, J=$ $5.0 \mathrm{~Hz}, 1 \mathrm{H}), 7.41(\mathrm{t}, J=7.7 \mathrm{~Hz}, 1 \mathrm{H}), 7.56(\mathrm{t}, J=7.8 \mathrm{~Hz}, 1 \mathrm{H}), 7.60(\mathrm{~d}, J=14.4 \mathrm{~Hz}, 1 \mathrm{H}), 7.71$ (d, $J=8.2 \mathrm{~Hz}, 1 \mathrm{H}), 7.78(\mathrm{~d}, J=14.4 \mathrm{~Hz}, 1 \mathrm{H}), 8.08(\mathrm{~d}, J=8.2 \mathrm{~Hz}, 1 \mathrm{H}) .{ }^{13} \mathrm{C} \mathrm{NMR} \delta 109.8,114.8$, 
120.3, 120.5, 124.6, 125.2, 127.5, 127.8, 128.2, 131.3, 138.2, 146.1. Anal. Calcd for $\mathrm{C}_{12} \mathrm{H}_{9} \mathrm{~N}_{3} \mathrm{~S}$ : C, 63.41; H, 3.99; N, 18.49. Found: C, 63.65; H, 3.87; N, 17.89 .

1-[(E)-3-Methyl-1-butenyl]-1H-1,2,3-benzotriazole (6c). Colorless oil; yield, 60\%. ${ }^{1} \mathrm{H}$ NMR $\delta$ $1.21(\mathrm{~d}, J=6.9 \mathrm{~Hz}, 6 \mathrm{H}), 2.60-2.65(\mathrm{~m}, 1 \mathrm{H}), 6.51(\mathrm{dd}, J=14.4,6.9 \mathrm{~Hz}, 1 \mathrm{H}), 7.27(\mathrm{~d}, J=$ $14.1 \mathrm{~Hz}, 1 \mathrm{H}), 7.39(\mathrm{t}, J=7.5 \mathrm{~Hz}, 1 \mathrm{H}), 7.52(\mathrm{t}, J=6.6 \mathrm{~Hz}, 1 \mathrm{H}), 7.65(\mathrm{~d}, J=7.5 \mathrm{~Hz}, 1 \mathrm{H}), 8.07$ (d, $J=8.4 \mathrm{~Hz}, 1 \mathrm{H}) .{ }^{13} \mathrm{C}$ NMR $\delta 22.3,29.5,110.0,120.1,121.0,124.2,127.8,130.3,136.8,146.1$. Anal. Calcd for $\mathrm{C}_{11} \mathrm{H}_{13} \mathrm{~N}_{3}$ : C, 70.56; H, 7.00; N, 22.44. Found: C, 70.73; H, 7.16; N, 23.08 .

1-[(E)-1-Butenyl]-1H-1,2,3-benzotriazole (6d). Yellow oil; yield, 50\%. ${ }^{1} \mathrm{H}$ NMR $\delta 1.01(\mathrm{t}, J=$ $7.5 \mathrm{~Hz}, 3 \mathrm{H}$ ), 2.34 (qd, $J=7.5,1.5 \mathrm{~Hz}, 2 \mathrm{H}), 6.47$ (quintet, $J=7.2 \mathrm{~Hz}, 1 \mathrm{H}), 7.19-7.32(\mathrm{~m}, 2 \mathrm{H}$ ), 7.40-7.45 (m, 1H), $7.57(\mathrm{~d}, J=8.4 \mathrm{~Hz}, 1 \mathrm{H}), 7.96-8.00(\mathrm{~m}, 1 \mathrm{H}) .{ }^{13} \mathrm{C}$ NMR $\delta 13.4,23.3,110.0$, 119.6, 122.3, 124.0, 127.6, 131.1, 132.8, 145.1. Anal. Calcd for $\mathrm{C}_{10} \mathrm{H}_{11} \mathrm{~N}_{3}$ : C, 69.34; $\mathrm{H}, 6.40 ; \mathrm{N}$, 24.26. Found: C, 69.47; H, 6.58; N, 23.87.

\section{Procedure for the preparation of 1,2-dibromo-benzotriazolyl alkanes 7a-d}

To a solution of vinylbenzotriazole $6(5 \mathrm{mmol})$ in dry dichloromethane was added bromine $(0.26 \mathrm{~mL}$, $5 \mathrm{mmol}$ ) at $0^{\circ} \mathrm{C}$. The mixture was maintained at this temperature for $30 \mathrm{~min}$ and then was allowed to warm up to room temperature. The mixture was stirred for $2 \mathrm{~h}$ and dichloromethane was evaporated under vacuum to give 1,2-dibromo-benzotriazolyl alkanes 7 in quantitative yield which can be used directly in the next step without further purification.

1-(1,2-Dibromo-2-phenylethyl)-1H-1,2,3-benzotriazole (7a). Light yellow solid; mp 74-75 ${ }^{\circ}$; yield, 100\%. ${ }^{1} \mathrm{H}$ NMR $\delta 6.21(\mathrm{~d}, J=11.4 \mathrm{~Hz}, 1 \mathrm{H}), 7.22(\mathrm{~d}, J=11.4 \mathrm{~Hz}, 1 \mathrm{H}), 7.44-7.53(\mathrm{~m}, 4 \mathrm{H})$, 7.60-7.69 (m, 3H), $7.72(\mathrm{~d}, J=8.4 \mathrm{~Hz}, 1 \mathrm{H}), 8.18(\mathrm{~d}, J=8.4 \mathrm{~Hz}, 1 \mathrm{H}) .{ }^{13} \mathrm{C} \mathrm{NMR} \delta 52.8,60.9$, $110.0,120.9,125.1,128.2,128.7,129.2,129.8,131.7,137.5,146.6$.

1-[1,2-Dibromo-2-(3-thienyl)ethyl]-1H-1,2,3-benzotriazole (7b). This compound was obtained as a mixture of isomers. Yellow powder; mp $120-121{ }^{\circ} \mathrm{C}$; yield, $96 \%$. ${ }^{1} \mathrm{H}$ NMR $\delta 6.99(\mathrm{~d}, J=$ $3.9 \mathrm{~Hz}, 1 \mathrm{H}), 7.07(\mathrm{~d}, J=3.9 \mathrm{~Hz}, 1 \mathrm{H}), 7.10-7.14(\mathrm{~m}, 1 \mathrm{H}), 7.26(\mathrm{~d}, J=3.9 \mathrm{~Hz}, 1 \mathrm{H}), 7.35(\mathrm{~d}, J=$ $3.9 \mathrm{~Hz}, 1 \mathrm{H}), 7.47-7.89(\mathrm{~m}, 3 \mathrm{H}), 8.17(\mathrm{~d}, J=8.1 \mathrm{~Hz}, 1 \mathrm{H}) .{ }^{13} \mathrm{C}$ NMR $\delta 109.8,109.9,112.7,114.1$, $114.9,120.4,120.5,120.8,124.7,124.8,125.3,127.7,127.8,127.9,128.3,128.5,130.8,131.4$, $139.9,146.2$.

1-(1,2-Dibromo-3-methylbutyl)-1H-1,2,3-benzotriazole (7c). Light yellow solid; mp 66-67 ${ }^{\circ} \mathrm{C}$; yield, 100\%. ${ }^{1} \mathrm{H}$ NMR $\delta 1.10(\mathrm{~d}, J=6.3 \mathrm{~Hz}, 3 \mathrm{H}), 1.21(\mathrm{~d}, J=6.6 \mathrm{~Hz}, 3 \mathrm{H}), 2.69(\mathrm{dh}, J=6.3$, $2.2 \mathrm{~Hz}, 1 \mathrm{H}), 5.27(\mathrm{dd}, J=9.9,2.1 \mathrm{~Hz}, 1 \mathrm{H}), 6.92(\mathrm{~d}, J=11.1 \mathrm{~Hz}, 1 \mathrm{H}), 7.61(\mathrm{t}, J=7.5 \mathrm{~Hz}, 1 \mathrm{H})$, $7.32(\mathrm{t}, J=7.5 \mathrm{~Hz}, 1 \mathrm{H}), 7.88(\mathrm{~d}, J=8.1 \mathrm{~Hz}, 1 \mathrm{H}), 8.19(\mathrm{~d}, J=8.4 \mathrm{~Hz}, 1 \mathrm{H}) .{ }^{13} \mathrm{C}$ NMR $\delta 15.1$, $15.9,22.2$, 30.6, 60.3, 62.6, 63.2, 65.1, 103.2, 110.7, 110.8, 119.4, 120.0, 125.9, 126.5, 129.2, 129.5, 131.7, 143.7. Anal. Calcd for $\mathrm{C}_{11} \mathrm{H}_{13} \mathrm{~N}_{3}$ : C, 38.07; H, 3.78; N, 12.11. Found: C, 37.85; H, $3.41 ; \mathrm{N}, 11.74$.

1-(1,2-Dibromobutyl)-1H-1,2,3-benzotriazole (7d). Brown oil; yield, 83\%. ${ }^{1} \mathrm{H}$ NMR $\delta 1.24(\mathrm{t}$, $J=7.2 \mathrm{~Hz}, 3 \mathrm{H}$ ), 2.17 (septet, $J=7.2 \mathrm{~Hz}, 1 \mathrm{H}), 2.55$ (sd, $J=7.2,2.7 \mathrm{~Hz}, 1 \mathrm{H}), 5.23$ (dt, $J=8.1$, $2.7 \mathrm{~Hz}, 1 \mathrm{H}), 6.89$ (d, $J=10.4 \mathrm{~Hz}, 1 \mathrm{H}), 7.46$ (t, $J=7.9 \mathrm{~Hz}, 1 \mathrm{H}), 7.61$ (t, $J=8.1 \mathrm{~Hz}, 2 \mathrm{H}), 7.70$ (d, $J=8.3 \mathrm{~Hz}, 1 \mathrm{H}), 8.13(\mathrm{~d}, J=8.4 \mathrm{~Hz}, 1 \mathrm{H}) .{ }^{13} \mathrm{C} \mathrm{NMR} \delta 10.5,29.3,55.6,61.0,110.1,120.4,125.0$, 
128.5, 146.0. Anal. Calcd for $\mathrm{C}_{10} \mathrm{H}_{11} \mathrm{Br}_{2} \mathrm{~N}_{3}$ : C, 36.07; H, 3.33; N, 12.62. Found: C, 36.22; H, $3.34 ; \mathrm{N}, 12.31$.

\section{Procedure for the preparation of amides 8a-r}

To a solution of 1,2-dibromo-benzotriazolyl alkane 7 (1 mmol) dissolved in $\mathrm{DMF} / \mathrm{H}_{2} \mathrm{O}$ $(3 \mathrm{~mL} / 1 \mathrm{~mL})$ was added amine $(5 \mathrm{mmol})$. The mixture was heated at $80{ }^{\circ} \mathrm{C}$ for $4 \mathrm{~h}$ till the starting material disappeared. Ethyl acetate $(30 \mathrm{~mL})$ was added and the diluted mixture was washed with water $(3 \times 10 \mathrm{~mL})$ followed by $1 \mathrm{M} \mathrm{NaOH}(10 \mathrm{~mL})$. The organic phase was dried over anhydrous $\mathrm{MgSO}_{4}$, filtered and concentrated in vacuo. The crude product was triturated in ether and filtered to give the desired amide $\mathbf{8}$.

$N$-Phenethyl-2-phenylacetamide (8a). Pale yellow solid; mp 98-100 ${ }^{\circ} \mathrm{C}$ ( $\mathrm{Lit}^{6} \mathrm{mp} 94-96{ }^{\circ} \mathrm{C}$ ); yield, 47\%. ${ }^{1} \mathrm{H}$ NMR $\delta 2.71(\mathrm{t}, J=6.6 \mathrm{~Hz}, 2 \mathrm{H}), 3.44(\mathrm{q}, J=6.9 \mathrm{~Hz}, 2 \mathrm{H}), 3.51(\mathrm{~s}, 2 \mathrm{H}), 5.50$ (br s, $1 \mathrm{H}), 7.19(\mathrm{~d}, J=6.0 \mathrm{~Hz}, 2 \mathrm{H}), 7.15-7.31(\mathrm{~m}, 8 \mathrm{H}) .{ }^{13} \mathrm{C}$ NMR $\delta 35.4,40.6,43.7,126.3,127.2$, $128.5,128.6,128.9,129.3,134.7,138.6,170.8$.

N-Butyl-2-phenylacetamide (8b). Yellow solid; mp $43-45{ }^{\circ} \mathrm{C}\left(\mathrm{Lit}^{7} \mathrm{mp} 49-51{ }^{\circ} \mathrm{C}\right)$; yield, 58\%. ${ }^{1} \mathrm{H}$ NMR $\delta 0.87(\mathrm{t}, J=6.9 \mathrm{~Hz}, 3 \mathrm{H}$ ), 1.25 (sextet, $J=7.5 \mathrm{~Hz}, 2 \mathrm{H}$ ), 1.40 (quintet, $J=7.8 \mathrm{~Hz}, 2 \mathrm{H}$ ), 3.29 (q, $J=6.9 \mathrm{~Hz}, 2 \mathrm{H}), 3.55$ (s, 2H), 5.66 (br s, 1H), 7.24-7.34 (m, 5H). ${ }^{13} \mathrm{C}$ NMR $\delta 13.6,19.8$, 31.4, 39.3, 43.7, 127.1, 128.8, 129.3, 135.0, 170.9 .

2-Phenyl-1-piperidino-1-ethanone (8c). Yellow solid; mp 82-83 ${ }^{\circ} \mathrm{C}\left(\mathrm{Lit}^{8} \mathrm{mp} 84-86{ }^{\circ} \mathrm{C}\right)$; yield, $61 \% .{ }^{1} \mathrm{H}$ NMR $\delta 1.31-1.37(\mathrm{~m}, 2 \mathrm{H}), 1.51-1.61(\mathrm{~m}, 4 \mathrm{H}), 3.37(\mathrm{t}, J=5.7 \mathrm{~Hz}, 2 \mathrm{H}), 3.58(\mathrm{t}, J=$ $4.8 \mathrm{~Hz}, 2 \mathrm{H}), 3.74(\mathrm{~s}, 2 \mathrm{H}), 7.22-7.34(\mathrm{~m}, 5 \mathrm{H}) .{ }^{13} \mathrm{C} \mathrm{NMR} \delta 24.3,25.4,26.1,41.1,42.8,47.2$, $126.6,128.5,128.6,135.3,169.3$.

N,2-Diphenylacetamide (8d). White powder; mp $117{ }^{\circ} \mathrm{C}\left(\mathrm{Lit}^{6} \mathrm{mp} 117-119{ }^{\circ} \mathrm{C}\right)$; yield, $72 \% .{ }^{1} \mathrm{H}$ NMR $\delta 3.72(\mathrm{~s}, 2 \mathrm{H}), 7.08-7.43(\mathrm{~m}, 11 \mathrm{H}) ;{ }^{13} \mathrm{C}$ NMR $\delta 44.8,119.9,124.5,127.6,128.9,129.2$, $129.5,134.5,137.6,169.3$.

$\mathrm{N}$-(tert-Butyl)-2-phenylacetamide (8e). Yellow powder; mp $113-115{ }^{\circ} \mathrm{C}\left(\mathrm{Lit}^{9} \mathrm{mp} 114-115{ }^{\circ} \mathrm{C}\right)$; yield, 68\%. ${ }^{1} \mathrm{H}$ NMR $\delta 1.28(\mathrm{~s}, 9 \mathrm{H}), 3.48(\mathrm{~s}, 2 \mathrm{H}), 5.20(\mathrm{br} \mathrm{s}, 1 \mathrm{H}), 7.23-7.37(\mathrm{~m}, 5 \mathrm{H}) .{ }^{13} \mathrm{C} \mathrm{NMR} \delta$ 28.6(3C), 44.8, 51.2, 127.1, 128.7, 128.9, 129.2, 129.3, 135.6, 170.4.

2-Phenyl- $\mathrm{N}$-(2-pyridinyl)acetamide (8f). Orange crystals; mp 93-95 ${ }^{\circ} \mathrm{C}\left(\mathrm{Lit}^{10} \mathrm{mp} 124-125{ }^{\circ} \mathrm{C}\right)$; yield, 85\%. ${ }^{1} \mathrm{H}$ NMR $\delta 3.71$ (s, 2H), 6.99 (td, $\left.J=7.0,2.0 \mathrm{~Hz}, 1 \mathrm{H}\right), 7.24-7.33$ (m, 5H), 7.66 (td, $J=7.0,2.0 \mathrm{~Hz}$, 1H), 8.20-8.27 (m, 2H), 9.24 (br s, 1H). ${ }^{13} \mathrm{C}$ NMR $\delta 44.3,114.3,119.6,127.2,128.7,129.1,134.0$, 138.3, 147.3, 151.5, 169.7. Anal. Calcd for $\mathrm{C}_{13} \mathrm{H}_{12} \mathrm{~N}_{2} \mathrm{O}$ : C, 73.57; H, 5.70; N, 13.20. Found: C, 73.54; H, 5.80; N, 13.08 .

2-Phenyl- $N$-(2-pyrimidinyl)acetamide (8g). White powder; mp $195{ }^{\circ} \mathrm{C}\left(\mathrm{Lit}^{11} \mathrm{mp} 193-196{ }^{\circ} \mathrm{C}\right)$; yield, 80\%. ${ }^{1} \mathrm{H}$ NMR $\delta 4.08(\mathrm{~s}, 2 \mathrm{H}), 6.99(\mathrm{t}, J=4.8 \mathrm{~Hz}, 1 \mathrm{H}), 7.27-7.36(\mathrm{~m}, 5 \mathrm{H}), 8.62(\mathrm{~d}, J=$ $4.8 \mathrm{~Hz}, 2 \mathrm{H}), 9.12$ (br s, $1 \mathrm{H}) ;{ }^{13} \mathrm{C}$ NMR $\delta 44.4,116.4,127.3,128.8,129.6,134.4,157.5,158.4$.

$N$-Phenethyl-2-(2-thienyl)acetamide (8h). Brown oil; yield, 52\%; ${ }^{1} \mathrm{H}$ NMR $\delta 2.74(\mathrm{t}, J=6.9$ $\mathrm{Hz}, 2 \mathrm{H}), 3.46$ (q, $J=6.9 \mathrm{~Hz}, 3 \mathrm{H}), 3.72(\mathrm{~s}, 2 \mathrm{H}), 5.76$ (br s, 1H), 6.83 (d, J=3.3 Hz, 1H), 6.95 (t, $J=5.4 \mathrm{~Hz}, 1 \mathrm{H}), 7.08(\mathrm{~d}, J=6.6 \mathrm{~Hz}, 1 \mathrm{H}), 7.19-7.28(\mathrm{~m}, 5 \mathrm{H}) .{ }^{13} \mathrm{C} \mathrm{NMR} \delta 35.4,37.5,40.8$, 
125.6, 126.5, 127.4 (2C), 127.5, 128.7 (2C), 136.0, 138.6, 169.9. HRMS calcd for $\mathrm{C}_{14} \mathrm{H}_{15} \mathrm{NOS}$ : 245.0874, found: 245.0870 .

$\boldsymbol{N}$-Butyl-2-(2-thienyl)acetamide (8i). Brown oil; yield, 92\%. ${ }^{1} \mathrm{H}$ NMR $\delta 0.89(\mathrm{t}, J=7.3 \mathrm{~Hz}$, $3 \mathrm{H}$ ), 1.27 (sextet, $J=7.0 \mathrm{~Hz}, 2 \mathrm{H}$ ), 1.40 (quintet, $J=7.2 \mathrm{~Hz}, 2 \mathrm{H}$ ), 3.22 (q, $J=7.2 \mathrm{~Hz}, 2 \mathrm{H}$ ), 3.78 (s, 2H), 5.67 (br s, 1H), 6.93-7.01 (m, 2H), 7.25 (dd, $J=5.1,1.2 \mathrm{~Hz}, 1 \mathrm{H}) .{ }^{13} \mathrm{C}$ NMR $\delta 13.7,19.9$, 31.4, 37.5, 39.5, 125.6, 127.3, 127.4 (2C), 136.3, 169.9. HRMS calcd for $\mathrm{C}_{10} \mathrm{H}_{15} \mathrm{NOS}$ : 197.0874 , found: 197.0876 .

1-Piperidino-2-(2-thienyl)-1-ethanone (8j). ${ }^{3}$ Brown oil; yield, 88\%. ${ }^{1} \mathrm{H}$ NMR $\delta 1.38-1.54(\mathrm{~m}$, $6 \mathrm{H}), 3.37$ (t, $J=5.8 \mathrm{~Hz}, 2 \mathrm{H}), 3.50(\mathrm{t}, J=5.8 \mathrm{~Hz}, 2 \mathrm{H}), 3.84(\mathrm{~s}, 2 \mathrm{H}), 6.82-6.88(\mathrm{~m}, 2 \mathrm{H}), 7.12$ (dd, $J=5.1,1.2 \mathrm{~Hz}, 1 \mathrm{H}) .{ }^{13} \mathrm{C}$ NMR $\delta 24.3,25.3,26.1,35.1,43.0,47.4,124.5,125.8,126.7,136.7$, 168.3.

1-Methyl-1-piperidin-1-yl-propan-1-one (8k). ${ }^{12}$ Yellow oil; yield, 50\%. ${ }^{1} \mathrm{H}$ NMR $\delta 0.97(\mathrm{~d}, J=$ $6.6 \mathrm{~Hz}, 6 \mathrm{H}), 1.23(\mathrm{~d}, J=6.6 \mathrm{~Hz}, 1 \mathrm{H}), 1.52-1.56(\mathrm{~m}, 6 \mathrm{H}), 2.20(\mathrm{~d}, J=6.6 \mathrm{~Hz}, 2 \mathrm{H}), 3.41(\mathrm{~m}, 2 \mathrm{H})$, 3.56 (m, 2H). ${ }^{13} \mathrm{C}$ NMR $\delta 17.9,22.6,24.4,25.5,25.7,26.5,42.0,42.4,46.8,109.1,119.9,123.9$, 127.7, 170.7 .

$N$-Butylbutanamide (8I). ${ }^{13}$ Colorless oil; yield, 82\%. ${ }^{1} \mathrm{H}$ NMR $\delta 0.88(\mathrm{t}, J=7.2 \mathrm{~Hz}, 3 \mathrm{H}), 0.91$ (t, $J=7.5 \mathrm{~Hz}, 3 \mathrm{H}$ ), 1.30 (sextet, $J=7.0 \mathrm{~Hz}, 2 \mathrm{H}$ ), 1.46 (quintet, $J=7.0 \mathrm{~Hz}, 2 \mathrm{H}$ ), 1.62 (sextet, $J=$ $7.0 \mathrm{~Hz}, 2 \mathrm{H}), 2.15(\mathrm{t}, J=7.3 \mathrm{~Hz}, 2 \mathrm{H}), 3.20(\mathrm{q}, J=6.9 \mathrm{~Hz}, 2 \mathrm{H}), 6.54$ (br s, $1 \mathrm{H}) .{ }^{13} \mathrm{C}$ NMR $\delta 13.5$ (2C), 19.1, 19.9, 31.4, 38.3, 39.1, 173.4. Anal. Calcd for $\mathrm{C}_{8} \mathrm{H}_{17} \mathrm{~N}_{1} \mathrm{O}$ : C, 67.09; H, 11.96; N, 9.78. Found: C, 66.82; H, 12.46; N, 9.65.

$\mathrm{N}$-[2-(Dimethylamino)ethyl]butanamide (8m). ${ }^{14}$ Colorless oil; yield, 32\%. ${ }^{1} \mathrm{H}$ NMR $\delta 0.87$ (t, $J=7.2 \mathrm{~Hz}, 3 \mathrm{H}), 1.58-1.62(\mathrm{~m}, 4 \mathrm{H}), 2.07(\mathrm{t}, J=7.8 \mathrm{~Hz}, 2 \mathrm{H}), 2.17(\mathrm{~s}, 6 \mathrm{H}), 2.31(\mathrm{t}, J=7.8 \mathrm{~Hz}$, 2H), 3.21-3.27 (m, 2H), 3.69 (br s, $1 \mathrm{H}) .{ }^{13} \mathrm{C}$ NMR $\delta$ 13.6, 18.9, 26.0, 38.5, 38.6, 45.0, 58.0, 173.0 .

1-Morpholino-1-butanone (8n). ${ }^{15}$ Colorless oil; yield, 50\%. ${ }^{1} \mathrm{H}$ NMR $\delta 0.97(\mathrm{t}, J=7.5 \mathrm{~Hz}, 3 \mathrm{H})$, 1.66 (sextet, $J=7.4 \mathrm{~Hz}, 2 \mathrm{H}), 2.31(\mathrm{t}, J=7.5 \mathrm{~Hz}, 2 \mathrm{H}), 3.47-3.50(\mathrm{~m}, 2 \mathrm{H}), 3.62-3.69(\mathrm{~m}, 6 \mathrm{H}) .{ }^{13} \mathrm{C}$ NMR $\delta 13.7,18.5,34.7,41.5,45.7,66.4,66.6,171.3$.

1-(4-Methylpiperazino)-1-butanone (8o). ${ }^{16}$ Colorless oil; yield, 62\%. ${ }^{1} \mathrm{H}$ NMR 0.97 (t, $J=7.3$ $\mathrm{Hz}, 3 \mathrm{H}$ ), 1.65 (sextet, $J=7.5 \mathrm{~Hz}, 2 \mathrm{H}), 2.31(\mathrm{~s}, 3 \mathrm{H}), 2.31$ (t, $J=7.5 \mathrm{~Hz}, 2 \mathrm{H}), 2.39$ (quintet, $J=$ $5.1 \mathrm{~Hz}, 2 \mathrm{H}), 3.50(\mathrm{t}, J=4.8 \mathrm{~Hz}, 2 \mathrm{H}), 3.63(\mathrm{t}, J=4.8 \mathrm{~Hz}, 2 \mathrm{H}) .{ }^{13} \mathrm{C}$ NMR $\delta 13.4,18.2,34.6,40.7$, 45.4, 54.2, 54.6, 170.9. Anal. Calcd for $\mathrm{C}_{9} \mathrm{H}_{18} \mathrm{~N}_{2}$ : C, 63.49; H, 10.66; N, 16.45. Found: C, 62.56; H, 10.97; N, 16.19 .

$\boldsymbol{N}$-Phenylbutanamide (8p). White powder; mp $82-84{ }^{\circ} \mathrm{C}\left(\mathrm{Lit}^{17} \mathrm{mp} 92{ }^{\circ} \mathrm{C}\right)$; yield, $95 \% .{ }^{1} \mathrm{H}$ NMR $\delta 0.98(\mathrm{t}, J=7.2 \mathrm{~Hz}, 3 \mathrm{H}), 1.74$ (quintet, $J=7.4 \mathrm{~Hz}, 2 \mathrm{H}), 2.32(\mathrm{t}, J=7.4 \mathrm{~Hz}, 2 \mathrm{H}), 7.08(\mathrm{t}, J=$ $7.4 \mathrm{~Hz}, 1 \mathrm{H}), 7.29(\mathrm{t}, J=7.8 \mathrm{~Hz}, 2 \mathrm{H}), 7.53(\mathrm{~d}, J=7.8 \mathrm{~Hz}, 2 \mathrm{H}), 7.70$ (br s, $1 \mathrm{H}) .{ }^{13} \mathrm{C}$ NMR $\delta 13.8$, 19.1, 39.6, 119.9, 124.2, 128.9, 138.1, 171.7. Anal. Calcd for $\mathrm{C}_{10} \mathrm{H}_{13} \mathrm{~N}_{1} \mathrm{O}: \mathrm{C}, 73.59 ; \mathrm{H}, 8.03 ; \mathrm{N}$, 8.58. Found: C, 73.49; H, 8.31; N, 8.60.

$\mathrm{N}$-(4-Pyridinyl)butanamide (8q). White powder; mp $136-138{ }^{\circ} \mathrm{C}$; yield, $95 \% .{ }^{1} \mathrm{H}$ NMR $\delta 0.99$ (t, $J=7.3 \mathrm{~Hz}, 3 \mathrm{H}), 1.75$ (sextet, $J=7.4 \mathrm{~Hz}, 2 \mathrm{H}), 2.38$ (t, $J=7.2 \mathrm{~Hz}, 2 \mathrm{H}), 7.54$ (d, $J=6.3 \mathrm{~Hz}$, 
2H), $8.46(\mathrm{~d}, J=6.3 \mathrm{~Hz}, 2 \mathrm{H}), 8.58$ (br s, $1 \mathrm{H}) .{ }^{13} \mathrm{C}$ NMR $\delta 13.6,18.7,39.5,113.6,145.6,150.3$, 172.5. Anal. Calcd for $\mathrm{C}_{9} \mathrm{H}_{12} \mathrm{~N}_{2}$ : C, 65.83; H, 7.37; N, 17.06. Found: C, 65.45; H, 7.66; N, 15.97 . $\mathrm{N}$-(1-Naphthyl)butanamide (8r). Violet powder; mp $113-115{ }^{\circ} \mathrm{C}$ (Lit ${ }^{18} \mathrm{mp} 120{ }^{\circ} \mathrm{C}$ ); yield, 68\%. ${ }^{1} \mathrm{H}$ NMR $\delta 1.05(\mathrm{t}, J=7.3 \mathrm{~Hz}, 3 \mathrm{H}), 1.82$ (quintet, $\left.J=7.3 \mathrm{~Hz}, 2 \mathrm{H}\right), 2.45(\mathrm{t}, J=7.3 \mathrm{~Hz}, 2 \mathrm{H}$ ), $7.43(\mathrm{t}, J=8.1 \mathrm{~Hz}, 1 \mathrm{H}), 7.46-7.50(\mathrm{~m}, 2 \mathrm{H}), 7.64$ (br s, 1H), 7.67 (d, J = 8.2 Hz, 1H), 7.81-7.87 $(\mathrm{m}, 3 \mathrm{H}) .{ }^{13} \mathrm{C}$ NMR $\delta 13.6,19.1,38.8,121.3,121.7,125.3,125.7,125.8,127.7,128.2,132.4$, 133.9, 172.5. Anal. Calcd for $\mathrm{C}_{14} \mathrm{H}_{15} \mathrm{~N}_{1} \mathrm{O}$ : C, 78.84; H, 7.09; N, 6.57. Found: C, 78.80; H, 7.24; $\mathrm{N}, 6.63$.

\section{References}

1. (a) Dinizo, S. E.; Freerksen, R. W.; Pabst, W. E.; Watt, D. S. J. Am. Chem. Soc. 1977, 99,182 and references cited therein. (b) Bascop, S-I.; Laronze, J-Y.; Sapi, J. ARKIVOC 2003, (v), 46.

2. Katritzky, A. R.; Toader, D.; Xie, L. Synthesis 1996, 1425.

3. Shen, W.; Kunzer, A. Org. Lett. 2002, 4, 1315.

4. Huh, D. H.; Jeong, J. S.; Lee, H. B.; Ryu, H.; Kim, Y. G. Tetrahedron 2002, 58, 9925.

5. Katritzky, A. R.; Offerman, R. J.; Cabildo, P.; Soleiman, M. Recl. Trav. Chim. Pays-Bas 1988, 107, 641.

6. Perreux, L.; Loupy, A.; Volatron, F. Tetrahedron 2002, 58, 2155.

7. McCarthy, D. G.; Hegarty, A. F. J. Chem. Soc., Perkin Trans. 2 1980, 579.

8. Wislicenus, W.; Erbe, R. Ann. Chem. 1920, 421, 119.

9. Baumgarten, H. E.; Chiang, N.-C. R.; Elia, V. J.; Beum, P. V. J. Org. Chem. 1985, 50, 5507.

10. Kato, T.; Yamamoto, Y.; Takeda, S. Yakugaku Zasshi 1973, 93, 1034.

11. Bream, J. B.; Lauener, H.; Picard, C. W.; Scholtysik, G.; White, T. G. Arzneim. -Forsch. (Drug Res.) 1975, 25, 1477.

12. Harada, R.; Kinoshita, Y. Bull. Chem. Soc. Jpn. 1967, 40, 2706.

13. De Benneville, P. L.; Levesque, C. L.; Exner, L. J.; Hertz, E. J. Org. Chem. 1956, 21, 1072.

14. Hromatka, O.; Kraupp, O.; Skopalik, C. Monatsh. Chem. 1953, 84, 349.

15. Medard, M. L. Bull. Soc. Chim. Fr. 1936, 5, 1343.

16. Millband, L. Chem. Abstr. 1968, 68, 105240k.

17. Schwartz, A. M.; Johnson, J. R. J. Am. Chem. Soc. 1931, 53, 1063.

18. Leete, E.; Juneau, K. N. J. Am. Chem. Soc. 1969, 91, 5614. 